\title{
RETOS PARA UNA EDUCACIÓN SOSTENIBLE E INCLUSIVA EN EL SIGLO XXI. EXPERIENCIAS SINGULARES
}

\author{
Oscar Kem-Mekah Kadzue \\ oscarkem@gmail.com \\ https://orcid.org/0000-0001-6684-5485 \\ Université de Yaoundé I, Camerún
}

Texto de la conferencia realizada en el marco del simposio "Miradas a la educación emergente en un contexto de cambios" desarrollado durante el I Congreso internacional de Ciencias sociales. II Encuentro de Socialización académica sobre experiencias investigativas en la Corporación Universitaria Autónoma de Nariño - extensión Santiago de Cali (Colombia) durante los días 13 y 14 de noviembre de 2020.

1. Retos que plantea el sistema educativo español de cara a la población afroespañola y migrante: educación inclusiva y antirracista como soluciones.

Cuando se habla de educación inclusiva, se presupone de entrada que se está en contexto de diversidad de cualquier índole (racial, religiosa, cultural, etc.). España es un ejemplo de esa diversidad de la que hablamos. El modelo educativo basado en la educación inclusiva pretende valorar la diversidad como elemento enriquecedor que permite el conocimiento y enriquecimiento mutuo, asimismo que la mejora de la calidad misma de la educación y, por ende, garantiza el desarrollo socioeconómico y cultural. Incluir a todos y todas en el proceso educativo sin importar el estatus social, origen, raza, religión o género y valorar las diferencias permite una verdadera y deseada globalización, en la que todos salen beneficiadores y la humanidad también.

El modelo inclusivo, tanto a nivel educativo como social, posibilita lo que Leopold Sédar Senghor (1971) llamó el gran encuentro del dar y recibir en el que "chaque ethnie, chaque nation, pourra apporter sa contribution. (...) seules y seront présentes, contribueront à bâtir la Civilisation de l'Universel». 
En este sentido, este modelo educativo se opone por ejemplo al modelo basado en la integración, ya que este último valora una cultura dominante, la hegemónica. Menosprecia y fagocita a las minoritarias y, por consiguiente, da lugar a fenómenos como la asimilación, la aculturación o el desarraigo cultural.

Es sorprendente pero, pese a las reformas educativas en materia de gestión de la diversidad (Ley Orgánica para la Mejora de la Calidad Educativa, 2013), en muchos países europeos, citamos a España y Francia entre otros, el modelo educativo seguido y defendido a toda costa tanto en el imaginario sociopolítico como entre algunos estudiosos en ciencias sociales es más bien el de la integración. Y cuando hablamos de integración, no nos referimos a integración justa desde un punto de vista bidireccional, sino desde un punto de vista unidireccional articulado con discursos supremacistas del tipo "ellos se tienen que integrar" o “ellos no se integran”. La verdadera pregunta para quienes piensan así sería: ¿y vosotros qué haces para integraros en el mundo que os rodea?, ¿es la integración una palabra para unos cuantos?

En realidad, el famoso "ellos" de "ellos tienen que integrarse" es una declaración de intenciones en la medida en que estigmatiza a la otredad dentro de la esfera de la periferia, la arrincona en la esfera de los no derecho habientes como diría Achille Mbembe, que por haber llegado o por vivir en un mundo que supuestamente no les pertenece, tienen el deber de olvidar sus individualidades idiosincráticas e integrarse en el supuesto mundo que les recibe. No obstante, es importante subrayar que en el imaginario sociopolítico de muchos países europeos, debido al borrón histórico que han asegurado las políticas educativas en relación con la presencia de las personas negras, que data de tiempos muy remotos debido a los antecedentes históricos entre África-Europa (Esclavitud y colonialismo), la población blanca (incluso buena parte de la gente culta) tiende a pensar que la presencia de las personas negras es un fenómeno reciente debido a los flujos migratorios contemporáneos.

La historia de millones de afroeuropeos queda pues relegada en los márgenes de la historia de la humanidad. Por eso, a día de hoy en la península ibérica, todavía es difícil que los españoles blancos vean a las 
personas negras como españoles a igual título que ellos. No lo conciben, no se lo imaginan, no se lo esperan porque en su imaginario España es blanca, España pertenece a las personas blancas y la presencia de la población negra en España es reciente y surge, en el mejor de los pensamientos, de la inmigración actual y, en el peor de los pensamientos de la inmigración clandestina. Así se percibe la población negra en España. De ahí la preponderancia de estas frases supremacistas dirigidas por algunas personas blancas, no solo los seguidores de la creciente extrema derecha, a las personas negras en España: "iQue se integren!", "No se integran”, “QQué bien hablas español!”, "Estás muy integrada, no eres como los demás", "Qué me vas a enseñar tú de la historia de España", "Vaya tela, conoces más la historia de mi país que yo", "Vete a tu país a quejarte", etc.

Gran parte de estas frases son recurrentes en la mayoría de producción literaria y artística de los afroespañoles e inmigrantes negroafricanos en España: Ser mujer negra en España (2019) de Desirée Bela Lobedee, Hija del camino (2020) de Lucia Mbomio, No es país para negras (2018) de Silvia Albert Sopale, Y tú por qué eres negro (2018) de Rubén Bermúdez, No hay país para negros (2018) de Kem-mekah Kadzue, Convergente, divergente o intruso (2017) de Edmundo Sepa, Cuando somos el enemigo (2019) de Jeffrey Abe Bans (Coord.), Mam'enying, cosas de la vida (2013) de Inongo Vi Makome, etc. No en vano el sociólogo y profesor asociado de Estudios Afroamericanos de la Universidad de California Stephen Small, entrevistado por Tania Adam (2019), advierte que "España no es un buen lugar para ser negro".

Añadiría que eso no es culpa de la población sino que es un fallo de las políticas educativas españolas que todavía carece de la mínima voluntad de fomentar una educación que fomente los principios de la educación inclusiva y sostenible. El sistema educativo español invisibiliza parte de su historia, la historia de millones de afroespañoles. La historia, los referentes y el pasado de gran parte de la población española ( $\mathrm{La}$ España negra) no se contemplan en los libros de historia y cultura. Es decir que la educación ni asegura la memoria histórica de los afroespañoles y ni cumple con el deber de reparación hacia la población 
negra en general. A continuación detallamos cómo se sustenta el racismo, el discurso del odio y la visión de los negros como les non ayant droits en España. Y nos reflexionamos asimismo sobre cómo desmontar ese pensamiento xenófobo a partir de una educación inclusiva y antirracista.

\subsection{El borrón histórico o blanqueamiento de la historia de España y sus consecuencias.}

La presencia negra o africana en general en España no es reciente y tampoco es fruto de las migraciones actuales como cree el imaginario social, sino que remonta a la edad media, esto es durante la ocupación musulmana de 711-1494. "Sabemos que en la edad media la presencia de gente negra, particularmente en las ciudades más comerciales, no era inusual; y que en el s. XII había peregrinos etíopes haciendo el camino de Santiago" (Canos Donnay, 2020). Seguidamente, después de la expulsión de los musulmanes en 1492, la presencia de personas africanas aumenta debido a la esclavitud. Según Cortés López (1986), citado por Antumi Toasije (2008), el Sur de la Península Ibérica abundaba en el siglo XV en personas esclavizadas norteafricanas y del África negra, siendo Sevilla el principal foco urbano de un tráfico que se originaba en Huelva.

Dicen los datos que a mediados del siglo XVI, la población negra suponía un 7,5\% del total de la población sevillana. Canos Donnay (2020) apunta en la misma línea que tanto cartagineses, como griegos y romanos que ocuparon España contaban con personas de origen subsahariano, tanto libres como esclavas, en sus filas. La esclavización de las personas negras, como en las Américas y otros países europeos siguió su ruta en España, permitiendo batir lo que se llamaba en aquellos tiempos el imperio español.

El historiador Antumi Toasije (2010) añade que incluso pese de haber sido abolida en el siglo XVIII, siguió practicándose en España hasta bien entrado en el siglo XIX. Esta presencia negra en España, que remonta de la edad media, sigue su curso durante la esclavización de las personas negras se refuerza a día de hoy gracias a los flujos migratorios, 
ha contribuido significativamente a lo que hoy es España y ha dejado un legado en todos los sectores. Un legado que las instituciones educativas no visibilizan ${ }^{1}$. Al tenor de lo dicho anteriormente, está claro que en España aquellos que sueltan discursos del odio hacia las personas de origen negra y musulmana desconocen la historia de lo que ellos consideran como su país, como si fuesen por derecho natural las únicas personas a las que les pertenece la Península Ibérica.

Nos podemos pues, plantear las preguntas siguientes: Cómo se va a tratar a la población negra en España con el respeto que se merece/cómo no va a crecer el complejo del salvador blanco (y cómo se va a empoderar la población negra y afrodescendiente en España) si... ...los jóvenes, de hoy que serán políticos mañana, son incultos y desconocen la historia de España ligada a la presencia negra.

...en la escuela hay un blanqueamiento de la historia de España.

...institucionalmente, prevalece una falta de reconocimiento y de memoria histórica hacia la comunidad negra.

...los jóvenes no saben que España esclavizó y que parte del legado actual de ayer y hoy viene de la esclavización de las personas negras desde el siglo XV hasta el XIX. Y se perpetúa a día de hoy con la ley de extranjería que condena a parte de la ciudadanía en la explotación en el sector agrario y de los cuidados.

...solo se les ocurren palabras como inmigración, muertes en el mediterráneo, al ver a las personas negras.

...desconocen a todos a los científicos, científicas e inventores afroespañoles y negros en general.

...no conocen a Juan Latino (1518-1596), Sor Teresa Juliana de Santo Domingo (1676-1748), Juan de Pareja (1606-1670), Elena o Eleno de Céspedes (1545-1588), Lucía Mbomio, Desirée Bela, Silvia Albert Sopale, Inongo, Donato Ndongo, Vi Makome, etc.

... apenas ha leído a Soyinka o Senghor, etc. (F. Iniesta, 2009).

\footnotetext{
${ }^{1}$ A nivel cultural, el documental Gurumbé: canciones de tu memoria negra deja constancia del impacto de los ritmos y melodías del África occidental en el flamenco y otras expresiones musicales de España.
} 
...desconocen el impacto de los ritmos y melodías del África occidental en el flamenco y otras expresiones musicales de la península.

...en el colegio no hay memoria histórica sobre el genocidio blanco sobre los negros, deconstrucción del discurso colonial y reparación.

...en la gran mayoría de los medios de comunicación, abundan un discurso estereotipado sobre el ser negro.

...las Áfricas siguen siendo las asignaturas pendientes del sistema educativo español.

...la educación no le da espacio a la descolonización de las mentes.

El blanqueamiento de la historia permite la enseñanza de una historia de España en la que solo existen protagonistas artistas, historiadores, políticos, científicos blancos. Este blanqueamiento de la historia en España ha contribuido al fortalecimiento de la supremacía blanca y, por ende el pensamiento según el que "para ser español, hay que ser blanco". De modo que en el imaginario popular, si una persona es de tez negra, no tiene derecho ni a opinar mal sobre España, tan solo debe darle las gracias por haberle acogido.

\subsection{Las Áfricas, asignaturas pendientes del sistema educativo español.}

Como apuntaba en un estudio anterior (Kem-mekah Kadzue, 2016), África sigue siendo la asignatura pendiente del sistema educativo español. La invisibilidad de lo afro (afroespañol/África) en la educación española no hace más que contribuir al incremento de los estereotipos y prejuicios tanto sobre África como sobre la población española de origen africana. En realidad, a la pregunta qué es lo primero que te viene a la mente cuando oyes la palabra "negros", "negras", "África", "africanos", "Africana" determinados jóvenes españoles contestarían: inmigración clandestina, valla, pateras, MENAS, disturbios sociales, guerras, hambre, gente necesitada, "ay pobres", tercer mundo. Y a la pregunta en qué fuente de información aprendiste esa visión sobre la otredad africana unos te dirían que en internet, en un programa de televisión, en la prensa, en el telediario, en el colegio. 
Como en el caso de los medios de comunicación, primero hay un falta de interés por África y, segundo, la poca información disponible en manuales contribuye más bien a pulir los estereotipos que los alumnos ya habían aprendido en los medios. L. Alonso (2000), mostrando el papel de la educación escolar en la construcción de la percepción estereotipada de África, afirma que en el:

“...manual, también de $2^{\circ}$ de la ESO, de la Editorial SM se "despacha" África en tres párrafos con el título de "África un continente olvidado": África es un continente con graves problemas políticos, étnicos, económicos y sociales, en algunos casos de tal envergadura que parecen tener muy difícil solución. La mayor parte de los países del África subsahariana son países empobrecidos y devastados por la guerra civil, la corrupción y los desastres, ya sean naturales o producidos por el hombre. Los frecuentes enfrentamientos entre grupos rivales siguen provocando números muertos (...) La ONU está destinando importantes fondos de ayuda al desarrollo" (p. 58).

En pocas palabras, se enseña al adolescente que África es sinónimo de desastre y caos. Podemos concluir que si los jóvenes españoles son unos ignorantes de la realidad africana, no es solo responsabilidad suya, también es la del sistema educativo. En una investigación anterior Kem-Mekah Kadzue (2012:79) en el que trabajamos con la juventud universitaria camerunesa y catalana como informantes, al colectivo de universitarios catalanes se le preguntó qué es lo que te han enseñado en la escuela/universidad sobre África.

Todos se quedaron siempre pensativos y a veces muy incómodos. Esta pregunta proporcionó el máximo grado de frases a medias y sin casi información. Podemos ilustrar lo dicho con el fragmento siguiente de un informante catalán: [Pensativa]... pues poca 
cosa. Tampoco no lo hemos visto. ¿Al nivel primaria o secundaria? Por ejemplo...[pensativa] bueno al nivel de la secundaria por ejemplo la parte de historia sí que nos han dicho que...[pensativa] pues el hecho...[pensativa] bueno el colonialismo sobre todo el hecho de la geometría o sea tampoco no hemos visto la parte esta ¿no? de los países económicamente más potentes en este sentido...[pensativa] bueno como... [pensativa] bueno ese provecho económico... [pensativa] o sea esa historia no lo hemos visto más, tal vez... [pensativa y luego nada]. (O. Kem-Mekah Kadzue, 2012:79). Sin embargo, algunos informantes catalanes dijeron sin rodeos, lo que en la escuela/universidad aprendieron de África: "muy poca cosa", "el expolio de las fuerzas extranjeras", "que es un continente pobre", "que es uno de los continentes más pobres del mundo", "pues básicamente que en África no hay agua, hay mucha hambre, la gente no tiene mucho dinero y todo esto"; y lo que más queremos destacar: "También sobre todo en mi escuela se me ha dicho que se necesita mucha cooperación, que África es el continente con más problemas y que sin embargo tiene muchos recursos que son explotados por esas empresas".

\subsection{Educación antirracista e inclusiva como solución.}

El problema de la educación antirracista en España es que mientras que las comunidades que sufren el racismo junto con un pugnado de aliados blancos llevan a cabo acciones para luchar contra él, parte de la ciudadanía española e incluso los que tienen el poder político, que sean de izquierda o de derecha, están ocupados en ofenderse y afirmar rotundamente que no hay racismo en España. Y después de esta negación sobre la existencia del racismo en España, suelen venir comentarios del tipo: "Si tan mal están aquí, pueden volver a sus países", "Ir a quejaros en vuestros países", etc. Las instituciones políticas y educativas no se han hecho cargo, de manera estructural y a nivel nacional, de la misión de luchar contra el racismo sistémico en el país. Pese a las recomendaciones de las Naciones Unidas a través el Decenio Internacional para los Afrodescendientes, que instan España a proteger los derechos de las personas de ascendencia africana, 
reconociendo sus aportaciones y la preservación de su rico patrimonio cultural.

Para una educación antirracista, es pues necesario que de entrada se reconozca el mal existente y que, por consiguiente, se lleve una verdadera política educativa antirracista para que la ciudadanía plantee una reflexión crítica sobre sus privilegios y su discurso y relación con los condenados de la tierra. Al nivel educativo, unos de los pasos de la educación inclusiva y antirracista serían:

a) Historia pasada y reciente de los afroespañoles: que el currículum educativo enseñe la historia de la España negra. Que haya un revisionismo histórico para que la ciudadanía deje de creer que España es solo blanca. Que las voces de figuras afroespañolas de ayer y hoy se contemplen en el currículo escolar. A continuación dejamos unos ejemplos:

- Juan de Pareja (1610-1670): Primer pintor negro de España.

- Elena o Eleno de Céspedes (1545-1588): Negra primera cirujana de la historia de España.

- Juan Latino (1594-1599): Primer catedrático negro de la historia de España.

- Primeros movimientos y Asociacionismos negros en España: las cofradías negras, como la de la San Benedicto de Parlermo en Granada (1501), la de Sant Jaume en Barcelona (1455), la de Nuestra Señora de los Ángeles en Sevilla (1554), o la Casa del Negres en Valencia (1472, etc.

b) África en el sistema educativo: que las Áfricas dejen de ser las asignaturas pendientes del sistema educativo español. Si no es así, los jóvenes de hoy que serán los políticos de mañana perpetuarán discursos del tipo: "África es una parte del mundo sin historia», "El negro representa al hombre natural en toda su barbarie y violencia" (Hegel), "Los negros no tienen historia" Heidegger (1934); África no tenía historia y "su drama es no haber entrado en la historia" Nicolás Sarkozy. 


\section{c) Esclavitud, colonialismo, neocolonialismo, reparación: que se} enseñe la historia de la esclavitud, colonialismo, saqueo e invasiones europeas de ayer y hoy en el continente Africano y en Hispanoamérica. Si no es así, los jóvenes de hoy que serán los políticos de mañana perpetuarán discursos del tipo: "Nosotros no colonizábamos, hacíamos una España más grande. ¿Qué otro país puede decir que un nuevo mundo fue descubierto por ellos?" Pablo Casado² (2018).

En la misma línea, citamos al ecuatoguineano Donato NdongoBidyogo (2009) que propone que se enseñe en las escuelas y en las universidades de España, de Europa, de América, la historia completa de África, porque debe formar parte de los conocimientos básicos de la humanidad, al igual que en África se estudia Historia Antigua o Medieval. Añade que es necesario que se sepa de dónde provienen el petróleo que mueve sus coches, alimenta sus industrias e ilumina calles y viviendas; conviene saber de dónde vienen el oro y los diamantes que se regala a los seres queridos el día de San Valentín, si no hay un solo yacimiento de esos minerales en Europa; se hace necesario saber de dónde sale y en qué condiciones se extrae el uranio que mueve las centrales nucleares que les dotan de energía; es bueno que sepan dónde compran el estaño, el manganeso, el cobalto, la tantalita, el cobre, el café, el cacao, la madera.

d) Segregación escolar: Que las políticas educativas luchen contra la segregación escolar que da lugar a la existencia en España de determinadas escuelas para personas de origen migrante. También debería replantearse el modelo de formación y la escolarización de los MENAS.

\footnotetext{
${ }^{2}$ Secretario General del PP.
} 
2. Retos que plantea el sistema educativo camerunés de cara a la identidad y empoderamiento: educación decolonial como solución.

Desde los soles de las independencias africanas, pocos son los países africanos donde las políticas educativas han diseñado un sistema educativo panafricanistas potente y acorde a las necesidades del fortalecimiento de la(s) identidades nacionales y por ende al empoderamiento del sujeto cultural africano. Es sabido que el período colonial supuso un quebrantamiento del sistema sociopolítico y cultural existente y una imposición de una cultura occidental, lo que ha dado lugar a una gran crisis identitaria en todos los países colonizados.

La novela Todo se desmorona (2010) de Chinua Achebe es un reflejo de esta realidad sociopolítica. El proceso de decolonización de la escuela y de las mentes sigue siendo un tema de actualidad hoy en día porque pese a haber celebrado sesenta años de independencia, en muchos países del África negra en general y de Camerún en particular, la crisis identitaria es aun patente. Para echar un vistazo en la realidad camerunesa, nos basamos en los resultados de entrevistas que hicimos en una investigación de hace unos años a ocho universitarios cameruneses sobre el tema de la crisis de identidad del sujeto cultural camerunés (2012).

De las entrevistas a cameruneses/camerunesas se desprendió una idea general en lo que respecta la percepción de la identidad propia camerunesa. Se trata de una identidad perjudicada por el contexto social en el que crece y permanece el joven camerunés, en la medida en que el reconocimiento y autoafirmación de la diversidad lingüística y cultural camerunesa por parte de la juventud vienen perjudicados por un contexto sociocultural que promueve más el conocimiento de las lenguas y culturas de los ex países colonizadores (Francia e Inglaterra) y occidentales en general (España, Alemania, Italia...) antes que lo propio camerunés.

Primeramente en el contexto familiar, muchas familias (los de las zonas urbanas principalmente) no usan la lengua materna como lengua vehicular en casa. Asimismo, no se inculca desde la niñez en la 
mente del joven el amor o el interés por lo propio. En la escuela sigue lo mismo dado que dependiendo de la pertenencia lingüística del alumnado (zona francófona o anglófona) el idioma vehicular en el contexto educativo puede ser el francés o el inglés. Además de que la educación viene dada en lengua extranjera (lenguas oficiales), los programas educativos todavía tienen una perspectiva colonial, pues es una educación heredada del modelo francés.

A este propósito, León Ngouo (1995:337), hablando de lo que él mismo llama "educación heredada" del modelo francés, advierte que: "Ni leur contenu, ni leurs objectifs ne sont adaptés aux besoins de l'Afrique, à sa culture, mais aux exigences du colonisateur". En realidad, desde la niñez (educación familiar) hasta la adolescencia (educación escolar) no se enseña a los niños a valorar lo propio, crecen con esta mentalidad de fuga, de culto a lo otro y además, por estar constantemente expuestos a los medios de comunicación y redes sociales, van imitando todo lo que perciben del modo de vida occidental.

$\mathrm{El} / \mathrm{la}$ joven camerunesa sufre de forma inconsciente una crisis identitaria desde muy pequeño. Todo lo que arriba hemos presentado forma parte de los condicionamientos que construyen la ideología estereotipada que los cameruneses tienen de sí mismos por estar orientada hacia la otredad occidental. En realidad, cuando somos niños no tenemos una capacidad de análisis y crítica propia, son los elementos como la educación familiar, escolar y los medios de comunicación los que nos los hacen desarrollar. Esta reflexión es válida porque la juventud camerunesa crece en un entorno donde la cultura propia es menos importante que la ajena y por eso aspira a vivirla.

Pero cuando crecen, cursan estudios como nuestros informantes, tienen un espíritu crítico y su proceso de cognición, en este caso la interfaz cognitiva entre discurso social y capacidad de reflexión propia, les da pensamiento crítico y se dan cuenta de que no tienen una identidad propia o, a lo mejor, que su sociedad va perdiendo valores que le son propios. En este contexto, podemos entender comentarios como los de una informante camerunesa: "Lo que es cierto es que es culpa de los padres, cada joven, padre de mañana deberá tenerlo en cuenta, la 
lengua materna es nuestra raíz, es lo que nos une a nuestra tierra, a nuestra cultura. No soy una González ${ }^{3}$, ni una Leticia de Lourdes".

En realidad, todos/as los/as informantes cameruneses mencionaron tener familiares que no hablan su lengua materna o mejor dicho no la valoran positivamente como algo propio. Eso se puede apreciar en los siguientes fragmentos del corpus de las entrevistas realizadas:

"Muchos de mis hermanos no pueden hablar correctamente la lengua materna a causa de las lenguas oficiales"; “(...) queremos vestirnos como los blancos"; "yo tengo sobrinas y primas que me dicen 'soy francesa' y le pregunto 'por qué me dices esto', 'porque hablo francés'. Hemos llegado a un punto donde hay gente que te dicen mi lengua es mala, mi lengua no es buena para escuchar, es difícil, no la valoran"; (Informante 1).

"En la casa de mi sobrino, sus hijos sólo hablaban el francés, siempre hablaban en francés porque no conocían ni una palabra en la lengua materna. Los medios de comunicación perjudican mucho la identidad de los cameruneses. Porque los niños ven en la tele cómo los europeos se visten y lo que quieren hacer es imitar"; (Informante 2).

"Hay también algunos que tienen vergüenza de su lengua y esto es lo peor que alguien pueda tener, tener vergüenza de su lengua es como tener vergüenza de sí mismo (mucha desolación en el rostro de la entrevistada). Es una pérdida de su identidad." (Informante 3)

En la misma línea, en una investigación realizada por Ndibnu Messina Ethé (2013), se comprueba que 70,5\% de los informantes no saben hablar ni escribir su lengua materna, como se puede apreciar en la tabla que viene a continuación.

\footnotetext{
${ }^{3}$ Esta referencia se relaciona con la lengua española que la entrevistada dice dominarla mejor que su lengua materna. Ella misma lamenta esto y se indigna por lo anormal que es esta situación. Y por lo tanto se indigna diciendo "no soy una González (nombre español) pero una Camerunesa. Y normalmente esto debería notarse en mi manera de hablar mi lengua materna". Pero desafortunadamente cuando habla en su lengua materna sus hermanos se burlan de ella porque no tiene el acento. Ella habla como una francesa.
} 


\begin{tabular}{|c|c|c|}
\hline $\begin{array}{l}\text { Ciudades } \\
\text { (Números de } \\
\text { informantes: } 400 \text {, de los } \\
\text { cuales } 300 \text { de la escuela } \\
\text { primera y } 100 \text { de la } \\
\text { secundaria) }\end{array}$ & $\begin{array}{l}\text { Porcentaje de los } \\
\text { alumnos } \\
\text { Que hablan y escriben } \\
\text { su lengua materna }\end{array}$ & $\begin{array}{l}\text { Porcentaje de los } \\
\text { alumnos } \\
\text { Que no hablan ni } \\
\text { escriben su lengua } \\
\text { materna }\end{array}$ \\
\hline Yaunde & $28,75 \%$ & $71,25 \%$ \\
\hline Duala & $30,25 \%$ & $69,75 \%$ \\
\hline Media & $29,5 \%$ & $70,5 \%$ \\
\hline
\end{tabular}

Pues, puede decirse que la diversidad lingüística y cultural de la que tanto se habla, "Camerún, África en miniatura", es una riqueza que corre un peligro. ¿Vamos hacia una pérdida de esta riqueza? Creemos que la respuesta es afirmativa porque la lengua que no se usa está condenada a desaparecer. Medidas de planificación lingüística necesitan ser tomadas. Mientras tanto se debe valorar positivamente que en 2010 el Ministerio de Enseñanza Secundaria creó el Departamento de Enseñanza de Lenguas Nacionales en la Escuela Normal Superior de Camerún con el fin de formar a futuros docentes de lenguas nacionales. De la percepción que tengamos de nosotros mismos dependerá la forma en que veamos al otro y seamos vistos.

Reforzar el sentimiento de identidad nacional es especialmente importante porque estamos muy expuestos a medios de comunicación que nos 'venden' Europa como algo que no tenemos y debemos desear. Como dice un informante camerunés, la consecuencia triste es que tenemos a “(...) jóvenes que tienen que buscar facilidades para ir a Europa porque en la tele se muestra todo un Eldorado, un lugar donde hay habitaciones que tocan casi el cielo. Todo el mundo tiene ese ideal y menosprecia su cultura, que considera de pobre, el país con dificultades económicas, (...)".

La concepción triste e ilusoria de Europa la podemos destacar en los extractos siguientes de las entrevistas a cameruneses: “A mi parecer, la gente de Camerún, como la de los demás países africanos ve España y Europa como el Eldorado. Para ellos, es allá donde se encuentran las buenas escuelas, las buenas universidades, buena gente, 
etcétera, breve, buena vida. Todos los pensamientos convergen hacia Europa". En la misma línea, Ambata (2018:49), apunta que "l'école postcoloniale est un vecteur d'hemorragie migratoire. Par sa nature, ses caractéristiques, ses modalités de fonctionnement et les conséquences d'ordres culturelles qu'elle charrie, tout, dans l'école postcoloniale, conduit la jeunesse de l'Afrique subsaharienne à quitter son terroir. Parlant de sa nature, l'école postcoloniale est une création de la colonisation qui est encore en vigueur après les indépendances, à la faveur du néocolonialisme».

La crisis identitaria de la que hablamos no solo se observa a nivel lingüístico sino también a nivel de los valores religiosos, el autoestima o la mirada que el ser negroafricano tiene sobre sí mismo, sobre su ser: consideración negativa sobre el color de piel, su pelo, etc. Eso da lugar pues a una generación de personas que sufren de un trauma inconsciente, que por consiguiente, aspiran a blanquearse la piel, llevar extensiones o pelucas de pelo liso a lo occidental sobre la cabeza, cuidarlo más que su propio pelo. Esta realidad puede comprobarse en estos fragmentos de la novela "No hay país para negros" (2018) en los que la protagonista hablar de la crisis identitaria en la zona urbana del país:

"Lamentaron también el hecho de que espiritualmente hablando, mucha gente desconocía valores y pilares de las creencias típicas africanas, las mismas que practican lod Kadzue, relativas al culto de los ancestros y el antropomorfismo de la naturaleza. Solo prevalecían creencias religiosas de origen colonial, la católica notablemente y otras tipologías de religión como:la protestante, los testigos de jehovás, la adventista (...).” (p. 41).

"También contaron a sus padres que en la capital las chicas se creían más bonitas por llevar extensiones en el pelo y capas de pintura en la cara. Prevalecía el parecer sobre el ser. Muchas chicas se aclaran la piel. No están orgullosas de su 
piel de ébano. Para ellas, el pelo liso a lo europeo es la referencia. Sus referentes de belleza son las cantantes y actores occidentales y americanos. ¿Cómo no van a pensar así si en los medios de comunicación y en el sistema educativo no se vende la negritud?” (p. 42).

De ahí que se hace necesario apremiante de descolonizar las mentes y potenciar el concepto de negritud, es decir, «la simple reconnaissance du fait d'être noir, et l'acceptation de ce fait, de notre destin de noir, de notre histoire et de notre culture» (Cesaire, 2000:92), tanto en la escuela como dentro de las familias. Es fundamental un modelo educativo que valore lo propio camerunés por encima de todo, porque de ello depende el florecimiento de una generación de cameruneses empoderados y orgullosos de su patrimonio cultural. Y finalmente de ello depende el desarrollo de África en miniatura, nuestra bonita nación.

Al tenor de todo lo que hemos comentado en esta ponencia, tanto respecto al sistema educativo camerunés como al sistema educativo español, puede concluirse que mientras los retos de los que hemos hablado no sean tratados, no se garantizará unas de las misiones imprescindibles de la educación recogidas en la declaración universal de derechos humanos. Nos referimos a aquella misión que apunta que "l'éducation doit viser au plein épanouissement de la personnalité humaine et au renforcement du respect des droits de l'homme et des libertés fondamentales».

\section{Referencias bibliográficas.}

Adam, T. (2019) "España no es un buen lugar para ser negro". Recuperado de: https://ctxt.es/es/20190821/Politica/27713/stephen-smalleuropa-negra-racismo-blanquitud-defensiva-tania-adam.html Ambata, L. (2018). L'école postcoloniale, vecteur d'hémorragies migratoires. En Manga, A. (2018). Éducation postcoloniale au 
Cameroun: enjeux et perspectives. Douala: Éditions Cheikh Anta Diop.

Antumi, T. (2008). La esclavitud en el XVI en territorios hispánicos. Brocar. Cuadernos de investigación histórica, 32, 99-116.

Antumi, T. (2010). La memoria y el reconocimiento de la comunidad africana y africano-descendiente negra en España: el papel de la vanguardia panafricanista. Nómadas. Revista Crítica de Ciencias Sociales y Jurídicas, 28(4), 1-41.

Canos, S. (2020). La historia de España también es negra. Recuperado de:

https://elpais.com/elpais/2020/09/28/africa_no_es_un_pais/ 1601314799_208238.html

Césaire, A. (2000). Cahier d'un retour au pays natal, Paris, Coll. «Poésie». Paris: Présence Africaine.

Cortés, J. (1986). Los orígenes de la esclavitud negra en España. Salamanca: Ediciones Universidad de Salamanca.

Kem-Mekah, O. (2012). Estereotipos y globalización, simetría y asimetría en la interacción cultural entre universitarios cameruneses y catalanes. Trabajo de grado. Universidad de Lleida, España.

Kem-Mekah, O. (2019). No hay país para negros. Barcelona: Editorial Wanafrica.

Manga, A. (2018). Éducation postcoloniale au Cameroun : enjeux et perspectives. Douala: Éditions Cheikh Anta Diop.

Ndibnu, J. (2012). Le français et les langues nationales (LN) au Cameroun: quelques considérations pédagogiques. Synergies Afrique des Grands Lacs, 2, 167-179.

Ndongo-Bidyogo, D. (2009). Acerca de los estereotipos sobre África. Castel y Sendín (Eds.). Imaginar África. Los estereotipos occidentales sobre África y los africanos, Madrid: Catarata.

Sédar, L. (1971). Liberté 2 : Nation et voie africaine du socialisme, discours. Conférences. Paris: Le Seuil. 